\title{
Application of Virtual Ant Algorithms in the Optimization of CFRP Shear Strengthened Precracked Structures
}

\author{
Xin-She Yang, Janet M. Lees, and Chris T. Morley \\ Department of Engineering, University of Cambridge \\ Trumpington Street, Cambridge CB2 1PZ, UK \\ xy227@eng.cam.ac.uk
}

\begin{abstract}
Many engineering applications often involve the minimization of objective functions. The optimization becomes very difficult when the objective functions are either unknown or do not have an explicit form. This is certainly the case in the strengthening of existing precracked reinforced concrete structures using external carbon fibre reinforced polymer (CFRP) reinforcement. For a given concrete structure, the identification of the optimum strengthening system is very important and difficult, and depends on many parameters including the extent and distribution of existing cracks, loading capacity, materials and environment. The choice of these parameters essentially forms a coupled problem of finite element analysis and parameter optimization with the aim of increasing the serviceability of the structure concerned. In this paper, virtual ant algorithms combined with nonlinear FE analysis are used in the optimization of the strengthening parameters. Simulations show that the location and orientation of the CFRP reinforcement has a significant influence on the behaviour of the strengthened structure. The orientation of the reinforcement with a fixed location becomes optimal if the reinforcing material is placed perpendicular to the existing crack direction. The implication for strengthening will also be presented.
\end{abstract}

\section{Introduction}

Nature inspired algorithms based on swarm intelligence and the self-organized behaviour of social insects can now be used to solve many complex problems such as the travelling salesman problem and the rerouting of traffic in a busy telecom network 5 . New algorithms are often developed in the form of a hybrid combination of biology-derived algorithms and conventional methods, and this is especially true in many engineering applications. On the other hand, the development of optimum strengthening and repair strategies for existing reinforced concrete structures is a challenging task. For a given structure, the most appropriate solution will depend on many factors such as the extent and distribution of any existing cracks, the loading capacity, the type of structure, available materials and environmental considerations [2,3,4]. The identification of the best strengthening method is essentially a coupled optimization problem consisting 
of finite element analysis and parameter optimization with the aim of increasing the serviceability of an existing structure. The optimization of parameters such as the area, spacing, location and orientation of additional CFRP reinforcing elements to strengthen a structure can be obtained by searching the parameter space and results from finite element (FE) analyses for a given set of these parameters. This paper aims to develop a simple optimization procedure to simulate different strengthening strategies for rectangular beams with dapped ends.

\section{Virtual Ant Algorithms}

Many problems in engineering and other disciplines involve optimizations that depend on a number of parameters, and the choice of these parameters affects the performance or objectives of the system concerned. The optimization target is often measured in terms of objective or fitness functions in qualitative models. The Virtual Ant Algorithm (VAA) starts with a troop of virtual ants, each ant randomly wonders in the phase space and in most cases; the phase space can be simply a 2-D or 3-D space. The main steps of the virtual ant algorithm are: 1) Create a initial population of virtual ants, and encode the function into virtual food; 2) Define the criterion for marking food/route with pheromone; 3) Evolution of virtual ants with time by random walking and broadcasting the best local to others if a better food location is found; 4) Evaluate the encoded concentration/locations of ants; 5) Decode the results to obtain the solution. Furthermore, in order to avoid the trapping at local maxima, a probability of $p=0.01 \sim 0.05$ is used to perturb the position and directions of the ants. There is a tradeoff between the computational efficiency in parameter searching and the computing time of finite element analysis. For the simulations used in this paper, the number of ants is taken to be in the range of 20-60.

The optimization problem in this paper forms a coupled problem including parameter-searching and the nonlinear finite element analysis. The virtual food in our optimization is the loading capacity. For each ant and each parameter set, a nonlinear finite element analysis is carried out in the sequential manner. The nonlinear finite element model used for our simulations is the smeared crack model developed by de Borst and others [1]. The difficulty is that the explicit form of the optimization function or load capacity is not known. Standard optimization approaches usually require a known optimization function before any optimization algorithms can be applied. However, we will show that virtual ant algorithms are also applicable to the functions that are not explicitly known.

\section{Simulations and Results}

\subsection{Strengthening of Beams with Dapped Ends}

The strengthening of reinforced concrete beams with dapped ends has been studied experimentally by Taher using various types of reinforcement [6]. Consider a beam of $2200 \times 300 \times 150 \mathrm{~mm}$ with dapped ends $(150 \mathrm{~mm} \times 150 \mathrm{~mm})$, 
where the reinforcement bars are placed at $100 \mathrm{~mm}$ from the top and $50 \mathrm{~mm}$ from the bottom surface of the beam. Both reinforcement layers have a total area of $100 \mathrm{~mm}^{2}$. The beam is loaded under the four-point bending conditions. The values used in the simulations for the concrete are, a Young's Modulus of elasticity $E_{\text {conc }}=21000 \mathrm{~N} / \mathrm{mm}^{2}$, a Poisson's ratio $\nu=0.15$, a compressive cube strength $f_{c u}=53 \mathrm{~N} / \mathrm{mm}^{2}$, and a tensile strength $f_{t}=6 \mathrm{~N} / \mathrm{mm}^{2}$. The additional CFRP strengthening reinforcement is considered as brittle elastic, but it is always within the elastic range for all the simulations. Figure 1 shows the crack stress at a load of $f=10 \mathrm{kN}$. We can see the stress concentration at the corners at both ends of the beam. This will lead to crack formation at these corners. If we strengthen this system with a CFRP reinforcing strap anchored near the

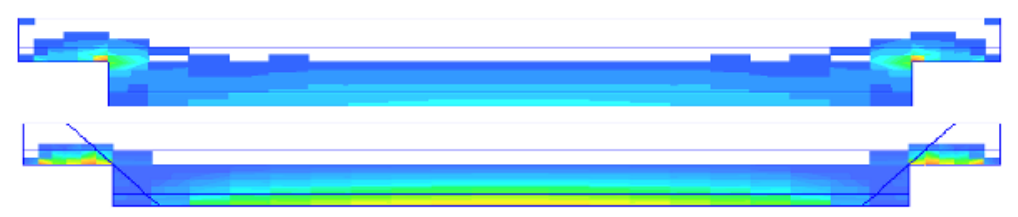

Fig. 1. Crack strain and effect of strengthening in a beam with dapped ends

top and bottom surfaces, we can study the effect of the strengthening system. The CFRP reinforcement is anchored at both ends but it is unbonded and not prestressed. The simulation of the strengthened beam gives the distribution of crack strains and crack patterns shown in the lower figure in Figure 1. We can see that the shear cracks are less extensive and have shifted away from the corners.

\subsection{Optimization of Strengthening Parameters}

The optimization of the strengthening parameters is carried out in the following manner. First, we use the same beam as shown in Figure 2 and vary the location $(x)$ of the midpoint and orientation $(\theta)$ of the CFRP reinforcement, then we run the FE analysis to obtain the final load. The load is then normalised using the failure load for $x / d=1$ and $\theta=0$. Figure 2 shows the load variation versus the orientation and location where the dots show the updating process of the parameter set $(x / d, \theta)$ and the fitness or load function which is the normalized final load where $d=300$ is the depth of the beam. From these figures, we can see that the final load reaches the maximum after about 60 parameter combinations, and this corresponds to the optimal angle of $\theta=45^{\circ}$. For a domain of $100 \times$ 100 grids, the virtual ant algorithms can reach the optimal setting (after only about 60 combinations) far more quickly than a regular searching algorithm where it would be necessary to consider 10000 possible combinations. The search efficiency is therefore increased by more than two orders or 125 times. Further work will extend the current work to the strengthening and repair of various structures under various time-dependent loading conditions and history. 

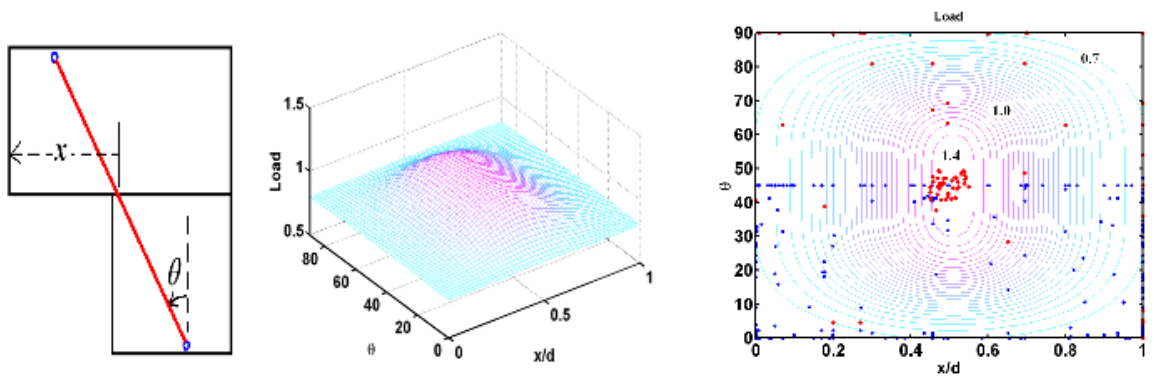

Fig. 2. Evolution of load with the location and orientation

\section{Conclusions}

By simulating the swarm interactions of social ants, we used the virtual ant algorithm to solve the function optimizations. An optimization problem for strengthening concrete structures is then formulated by coupling parameter searching and nonlinear finite element analysis. By using efficient parameter searching to optimize the search for the best strengthening parameters and combining it with FE simulations, we have reached the optimal load without knowing the shape of the fitness function. Simulations show that both the location and orientation of additional CFRP strengthening reinforcement elements are important. In the case of a beam with dapped ends, the optimal strengthening parameters are $x=0.5 d$ and $\theta=45^{\circ}$. As expected, this result suggests that the best option is to strengthen in the direction that is perpendicular to the direction of major cracks. Further research will consider the optimum strengthening strategy for structures with a complex geometry and time-dependent loading history.

Acknowledgement. The authors are grateful to EPSRC for their financial support (GR/S55101/01).

\section{References}

1. de Borst R.: Smeared cracking, plastity, creep and thermal loading - a unified approach, Comp. Meth. Appl. Mech. Eng., 62 (1987) 89-110.

2. Lees J. M., Winistoerfer A. U. and Meier, U.: External prestressed CFRP straps for the shear enhancement of concrete, ASCE J Composites for Construction, 6 (2002) 249-256.

3. Hoult N. A. and Lees J. M.: Shear retrofitting of reinfoced concrete beams using CFRP straps, Proc. Advanced Composite Materials in Bridges and Structures, 20-23 July, Calgary, (2004).

4. Lees, J.M., Morley, C.T., Yang, X.S., Hasan Dirar, S.M.O.: Fibre reinforced polymer strengthening of pre-cracked concrete structures, Concrete, 39 (2005) 36-37

5. Solnon C.: Ants can solve constraint satisfaction problems. IEEE Trans. Evolutionary Computing, 6 (2002) 347-357.

6. Taher, S. E. M.: Strengthening of critically designed girders with dapped ends, Structures and Buildings, 158 (2005) 141-152. 